Case report

\title{
Post dural puncture headache in a pediatric patient with idiopathic intracranial hypertension
}

\author{
OLUBUKOLA O. NAFIU MD FRCA*, DOMINIC \\ MONTEROSSO DO*, SARAH R. WALTON MD* \\ AND STUART BRADIN MD† \\ Departments of *Anesthesiology and +Emergency Medicine, University of Michigan, \\ Ann Arbor, MI, USA
}

\begin{abstract}
Summary
We describe the occurrence of postdural puncture headache (PPDH) in an adolescent with idiopathic intracranial hypertension (IIH) and its successful management with an epidural blood patch. PPDH is a very rare occurrence in patients with intracranial hypertension and is described as a paradoxical situation in the literature. There are only two previous case reports (in adults) of the possible association. A 15-year-old obese patient with a diagnosis of IIH had an uneventful diagnostic spinal tap using a $22 \mathrm{G}$ Quincke needle in the pediatric emergency department but returned $24 \mathrm{~h}$ later with PPDH. After a failed trial of conservative management, she had an uneventful but curative epidural blood patch with $15 \mathrm{ml}$ of autologous venous blood and was able to return to school the day after the blood patch. Followup review by her neuro-ophthalmologist shows resolution of her headaches, considerable improvement in her visual field defect and resolution of papilledema. This is the first report of PPDH and its successful management with an epidural blood patch in a pediatric patient with IIH.
\end{abstract}

Keywords: lumbar puncture; postdural puncture headache; pseudotumor cerebri; idiopathic intracranial hypertension; epidural blood patch

\section{Introduction}

Idiopathic intracranial hypertension (IIH) is a heterogeneous syndrome characterized by increased resting intracranial pressure without evidence of intracranial space-occupying lesion (1). It is also known as pseudotumor cerebri or benign intracranial hypertension, although the condition, if far from benign, because it

Correspondence to: Dr O.O. Nafiu, Department of Anesthesiology, University of Michigan, Room UH 1H247, 1500 East Medical Drive, Ann Arbor, MI 48109 0048, USA (email: onafiu@med.umich.edu). may cause in patients to suffer from incapacitating headaches, intractable nausea and impaired or loss of vision if they are not properly managed. Diagnosis of IIH is based on the modified Dandy criteria (2) which include clinical features of raised intracranial pressure without focal neurological signs (except visual field defect and abducens nerve palsy), normal cranial computed tomography (CT) or magnetic resonance imaging, cerebrospinal fluid (CSF) pressure $>250 \mathrm{~mm}$ $\mathrm{H}_{2} \mathrm{O}$ and normal CSF contents.

Idiopathic intracranial hypertension predominantly affects obese women of childbearing age (3). 
Although more common in women, IIH is well described in children and has pediatric-specific clinical profile (4-6). In both adult and pediatric patients with IIH a spinal tap is required for diagnosis and it may be therapeutic in some patients (7). Unfortunately the commonest complication of spinal tap in adults and children is postdural puncture headaches (PDPH) which may be incapacitating $(8,9)$.

Postdural puncture headache appears to be very uncommon in patients with IIH. These are a highrisk group of patients: a lumbar puncture (LP) is required in all cases and these LPs are often performed by neurologists and other physicians who routinely use large bore, cutting needles (10). The occurrence of PDPH in patients with IIH has been described as a paradoxical situation because one is a high-pressure headache state and the other a low CSF pressure headache condition. Some neurologists and neuro-ophthalmologists even believe the two conditions cannot occur in the same patient (11). Many neurology and neuroophthalmology textbooks and review articles do not discuss the possibility of the two conditions occurring in the same patient $(1,8,12,13)$. To the best of our knowledge there are only two reported cases (both adults) of PDPH in patients with IIH $(11,14)$. We report the first case of PDPH following diagnostic LP in a pediatric patient with pseudotumor cerebri who was successfully managed with an epidural blood patch.

\section{Case report}

A 15-year-old obese Caucasian adolescent was referred by her neuro-ophthalmologist to the pediatric emergency department (ED) for a head computed tomography (CT) to be followed by a spinal tap. The patient was apparently well until 4 weeks prior to presentation when she started having headaches. These were described as 'band like' in nature and localized in the fronto-parietal region but worse on the left side. The headaches were sometimes associated with photophobia and phonophobia. They were sometimes worse in the early mornings and occasionally caused her to miss school. There was no nausea or vomiting and no symptoms of intra- or paracranial infections. There is a positive family history of migraine headaches in the patient's father. She was not on any regular medications and her menstrual cycle had been regular since menarche at the age of 12 years.

The patient was initially reviewed by her primary care physician who made a diagnosis of migraine and commenced her on ibuprofen. However, there was only transient relief and she continued to have headaches every day despite medications. The parents became concerned when she began complaining of left retro-orbital pain and decreased peripheral vision worse when coughing or sneezing. She also had transient visual obscurations. The patient was referred to a neuro-ophthalmologist who noted that she had normal visual acuity, some peripheral visual constriction and bilateral papilledema. She was then referred to the pediatric ED. Apart from noting bilateral papilledema, her physical examination in the ED was normal. A noncontrast head CT was normal and we proceeded to perform an LP.

After informed consent, we placed the patient in the left lateral decubitus position and anesthetized the skin over the L3:L4 interspace. Under aseptic conditions, a spinal tap was performed using a 22G Quincke needle (the only available size and type in the pediatric 'spinal kit' in our hospital) with the bevel oriented parallel to the dura fibers. CSF opening pressure was $350 \mathrm{mmH}_{2} \mathrm{O}$, measured with the patient relaxed and leg extended in the lateral decubitus position. Closing pressure after removing $20 \mathrm{ml}$ of clear colorless CSF was $180 \mathrm{mmH}_{2} \mathrm{O}$ and the patient claimed some improvement in her headache. Laboratory analysis of the CSF was normal and the patient was discharged home with a prescription for acetazolamide, an appointment with a nutritionist for weight management and an exercise program, and to follow up with her neuro-ophthalmologist in 2 weeks.

She however presented to the pediatric ED $24 \mathrm{~h}$ later with a new type of headaches described as bilateral, occipital in location, worsened by the upright posture and relieved by lying down. These headaches were graded as worse than her 'usual' headaches and she was unable to stay upright long enough to go to school. There were no other symptoms and her physical examination was normal. We made a diagnosis of PPDH and offered the patient the choice of an epidural blood patch or the conservative approach of withholding acetazolamide, drinking liberal oral fluids, and ibuprofen. 
She chose the noninvasive option and promised to return if there was no improvement.

She returned to the ER the following day, still having classical PDPH hence the decision was made to proceed with a blood patch. Prior to performing the blood patch, a repeat cranial CT scan was carried out and this was normal. The procedure and risks were explained to the patient and her mother. Under strict asepsis and with the patient in the left lateral position, the epidural space was located at L3 : 4 using loss of resistance to saline technique. Following this, $15 \mathrm{ml}$ of venous autologous blood was slowly injected into the epidural space after which she noticed immediate improvement in her headaches. She was discharged home a few hours after the blood patch and was able to go to school the following day. There has been no recurrence of postural headaches since the blood patch and no reported complications from the procedure. Follow up with the neuro-ophthalmologist showed resolution of her papilledema and considerable improvement in visual field defect.

\section{Discussion}

To the best of our knowledge, this is the first reported case of PDPH in a pediatric patient with IIH. Our patient fulfilled the criteria for the diagnosis of IIH and required LP for diagnostic and therapeutic purposes (2). Patients with IIH often suffer from chronic, sometimes incapacitating headaches and in some the disease may impair vision or lead to blindness. LP is a diagnostic requirement and it may be therapeutic in some patients. Occurrence of PDPH (a low-pressure headache syndrome) in patients with IIH (a high-pressure headache disorder) is extremely rare (14).The two conditions occur at opposite ends of the intracranial pressure spectrum. Although the mechanism of IIH is unclear, experts believe that it results from abnormal CSF kinetics - an imbalance between production and absorption (1). This is the basis of using LP and CSF removal as a treatment modality with the goal of restoring normal CSF pressure. Some patients may require a lumboperitoneal shunt (14). The pathogenesis of PDPH is thought to result from CSF loss leading to reduced CSF volume and pressure. When the patient stands up, the intracranial contents sag, putting traction on pain-sensitive dura fibers and producing headache and sometimes neck pain $(8,9)$.
A 22G Quincke needle was used to perform the LP by the ED physicians in our patient. This is the needle used most commonly for LP by nonanesthetists in our institution. We agree with Kokki et al. (15) that PDPH is just as common in children as in adults when a $22 \mathrm{G}$ needle is used for diagnostic LP. However, it is hard to extrapolate their findings to patients with $\mathrm{IIH}$ as the goal in these patients is to reduce CSF volume and pressure. It would seem intuitive that increased CSF loss should not affect the incidence of PDPH in patients with IIH which is the currently popular belief. However, since the occurrence of PDPH in the present patient, we have instituted a joint prospective audit with the neurologists and ED physicians in our hospital. The goal is to describe patient characteristics and to specifically look for PDPH in patients presenting with IIH to our unit in the hope our findings would help to shed some light on the subject.

Our patient's CSF closing pressure was low $\left(180 \mathrm{mmH}_{2} \mathrm{O}\right)$ after removing $20 \mathrm{ml}$ of CSF which may have contributed to the occurrence of PDPH. However, the aim of LP and/or CSF drainage procedure in patients with IIH is reduction in CSF pressure and maintenance of this low pressure with a diuretic such as acetazolamide $(1,4)$. Dehydration could potentially worsen the symptoms in patients with PDPH which creates a therapeutic dilemma. We advised our patient to stop acetazolamide during the conservative management of her PDPH. She was willing to persevere with this approach for only $24 \mathrm{~h}$ before requesting a blood patch.

The role of epidural blood patch in patients with PDPH is well established and has become the 'gold standard' for the treatment of severe PDPH unresponsive to conservative management (16). Most experts agree that due to the high cure rate of epidural blood patch and the relative paucity of serious side effects, there is little reason to delay using it in patients with severe PDPH (9). A blood patch was curative in our patient. It is however impossible to make evidence-based recommendations for the use of epidural blood patch in patients with IIH as its use is limited to a handful of case reports $(11,14)$. Prior to performing the blood patch we were concerned about the theoretical possibility of worsening papilledema and visual function with restoration of normal/elevated CSF pressure. This has never being reported and did not occur in our patient. 
In conclusion, we report the first, well-documented occurrence of PDPH in an adolescent with IIH following LP and its successful treatment with an epidural blood patch. Like the two previous reports in adults, a low-pressure headache syndrome may occur in pediatric patients with IIH and may be successfully managed with an epidural blood patch. The possibility of PDPH should be considered in patients with IIH who develop postural headache following LP or CSF drainage procedure and an epidural blood patch could be therapeutic in these patients.

\section{References}

1 Friedman DI. Pseudotumor cerebri. Neurol Clin N Am 2004; 22: 99-131.

2 Ahlskog JE, O’Neill BP. Pseudotumor Cerebri. Ann Intern Med 1982; 97: 249-256.

3 Durcan FJ, Corbett JJ, Wall M. The incidence of pseudotumor cerebri. Population studies in Iowa and Louisiana. Arch Neurol 1988; 45: 875-877.

4 Lessell S. Pediatric pseudotumor cerebri (idiopathic intracranial hypertension). Surv Ophthalmol 1992; 37: 155-166.

5 Baker RS, Baumann RJ, Buncic JR. Idiopathic intracranial hypertension (pseudotumor cerebri) in pediatric patients. Pediatr Neurol 1989; 5: 5-11.

6 Cinciripini GS, Donahue S, Borchert MS. Idiopathic intracranial hypertension in prepubertal pediatric patients: char- acteristics, treatment, and outcome. Am J Ophthalmol 1999; 127: 178-182.

7 Soler D, Cox T, Bullock P. Diagnosis and management of benign intracranial hypertension. Arch Dis Child 1998; 78: 89-94.

8 Evans RW. Iatrogenic disorders: complications of lumbar puncture. Neurol Clin N Am 1998; 16: 83-105.

9 Wee LH, Lam F, Cranston JA. The incidence of post dural puncture headache in children. Anaesthesia 1996; 51: 1164-1166.

10 Serpell MG, Haldane GJ, Jamieson DR et al. Prevention of headache after lumbar puncture: questionnaire survey of neurologists and neurosurgeons in the United Kingdom. $\mathrm{Br}$ Med J 1998; 316: 1709-1710.

11 Lussos SA, Loeffler C. Epidural blood patch improves postdural puncture headache in a patient with benign intracranial hypertension. Reg Anesth 1993; 18: 315-317.

12 Gasser JS. Neuro-Ophthalmology, 2nd edn. Philadelphia: JB Lippincott, 1990.

13 Silberstein SD, Lipton RB, Dalessio DJ. Wolff's Headache and Other Facial Pain. New York: Oxford University Press, 2001.

14 Loh Y, Labutta RJ, Urban ES. Idiopathic intracranial hypertension and post-lumbar puncture headache. Headache: J Head Face Pain 2004; 44: 170-173.

15 Kokki H, Salonvaara M, Herrgard E et al. Post dural puncture headache is not an age related symptom in children: a prospective, open randomized, parallel group study comparing a 22-gauge Quincke with a 22-gauge Whitacre needle. Paediatr Anaesth 1999; 9: 429-434.

16 Ylonen P, Kokki H. Management of post dural puncture headache with epidural blood patch in children. Paediatr Anaesth 2002; 12: 525-528.

Accepted 5 September 2004 